\title{
Glucose Titration Studies in Patients with Chronic Progressive Renal Disease *
}

\author{
Richard E. Rieselbach, $†$ Stewart W. Shan kel, $\$$ Eduardo Slatopolskr, \\ Herbert Lubowitz, and Neal S. Bricker $\S$ \\ (From the Renal Division, Department of Internal Medicine, Washington University School \\ of Medicine, St. Louis, Mo.)
}

\begin{abstract}
Summary. Glucose titration studies were performed on 17 patients with either chronic pyelonephritis or chronic glomerulonephritis. Glomerular filtration rates for the group ranged from 4.3 to $58.1 \mathrm{ml}$ per minute. In none of the patients in whom the glomerular filtration rate was over $15 \mathrm{ml}$ per minute was there appreciable splay, and the mean titration curve for these patients resembled that obtained by Smith and associates in normal man (1). In half of this group of eight patients, GFR ranged from 16.6 to $22.7 \mathrm{ml}$ per minute; in the other half values ranged from 42.3 to $58.1 \mathrm{ml}$ per minute. Yet, the mean titration curves were identical for the two groups. In addition, no difference was observed in the titration curves for patients with pyelonephritis and those with glomerulonephritis. In patients with GFR values below 15 $\mathrm{ml}$ per minute, increased splay was observed, and below a GFR of $10 \mathrm{ml}$ per minute, the splay was very marked. Both the absence of exaggerated splay in patients with reduction of glomerular filtration rate by as much as $85 \%$, and the emergence of exaggerated splay in patients with more marked reduction of GFR, require explanation. Theoretical considerations are presented in the text.
\end{abstract}

\section{Introduction}

Glucose reabsorption by the mammalian kidney is characterized by a maximal rate of transport or a Tm. When the plasma glucose concentration is increased progressively by glucose infusion, the increment in the filtered glucose is reabsorbed quantitatively until the $\mathrm{Tm}$ is approached. Before $\mathrm{Tm}$ is reached, however, some glucose is excreted, and in man the load must exceed the Tm by ap-

* Submitted for publication June 27, 1966; accepted October 20, 1966.

Supported by U. S. Public Health research grant AM-09976, training grant AM-5248, and grant FR-36 from the General Clinical Research Centers Branch, Division of Research Facilities and Resources.

† Special Fellow, U. S. Public Health Service. Present address: Dept. of Medicine, University of Wisconsin, School of Medicine, Madison, Wisc.

$\ddagger$ Research Fellow, U. S. Public Health Service.

$\S$ Research Career Award, U. S. Public Health Service.

Address requests for reprints to Dr. Neal S. Bricker, Barnes and Wohl Hospitals, 660 S. Euclid Ave., St. Louis, Mo. 63110. proximately $50 \%$ before the reabsorptive capacity is saturated (1). Thus, when the filtered load is plotted against glucose reabsorption, splay appears in the curve as reabsorption approaches $\mathrm{Tm}$. In analyzing glucose titration curves to determine the distribution of glomerulotubular balance among the nephrons of normal individuals, Smith and his colleagues (1) obtained a bimodal frequency distribution curve. On the basis of this curve, it was suggested that the human kidney includes two groups of nephrons, one a large population, the other a relatively small population. If splay in the titration curve does indicate inhomogeneity of glomerulotubular balance for glucose, the glucose titration technique might lend itself well to an examination of the level of homogeneity of nephrons in the diseased kidney. If any segment of the nephron population exists in which tubular function (i.e., glucose reabsorption) is preferentially impaired, the ratio of GFR to glucose reabsorptive capacity should be greater than the average value for the whole kidney. Hence glucose should ap- 
TABLE I

Glomerular filtration rate, Tm $m_{\text {olucose }}, G F R / T m_{\text {glucose }}$ ratios, and clinical data for the 17 patients studied*

\begin{tabular}{|c|c|c|c|c|c|c|c|c|}
\hline Patient & Diagnosis & Age & Sex & BSA & GFR & $T m_{\text {glucose }}$ & GFR/Tm $\mathrm{m}_{\mathrm{g} \text { lucose }}$ & $\mathrm{PCr}$ \\
\hline & & years & & $m^{2}$ & $m l / m i n$ & $m g / m i n$ & & $m g / 100 m$ \\
\hline 1 & Chronic active glomerulonephritis $†$ & 18 & $\mathbf{M}$ & 1.55 & 58.1 & 242.2 & 0.24 & 1.7 \\
\hline 2 & Chronic glomerulonephritist & 18 & $\mathrm{~F}$ & 1.73 & 52.0 & 221.7 & 0.23 & 1.7 \\
\hline 3 & Chronic pyelonephritis & 59 & $\mathbf{F}$ & 1.60 & 43.8 & 285.0 & 0.15 & 1.1 \\
\hline 4 & Chronic glomerulonephritis $\dagger$ & 20 & $\mathrm{M}$ & 2.10 & 42.3 & 158.8 & 0.27 & 2.5 \\
\hline 5 & Chronic pyelonephritis & 41 & $\mathrm{~F}$ & 1.45 & 22.7 & 65.2 & 0.35 & 3.0 \\
\hline 6 & Chronic pyelonephritis & 66 & $\mathbf{M}$ & 1.80 & 17.8 & 108.0 & 0.16 & 3.9 \\
\hline 7 & Chronic glomerulonephritis & 56 & $\mathbf{M}$ & 1.73 & 17.2 & 45.3 & 0.38 & 4.3 \\
\hline 8 & Acute and chronic pyelonephritis $\dagger$ & 54 & $\mathrm{~F}$ & 1.55 & 15.0 & 62.2 & 0.24 & 2.6 \\
\hline 9 & Chronic glomerulonephritis $\dagger$ & 44 & $\mathbf{M}$ & 1.90 & 16.6 & 51.8 & 0.32 & 3.9 \\
\hline $9 \mathrm{a}$ & Chronic glomerulonephritis $\dagger$ & 44 & $\mathbf{M}$ & 1.90 & 13.9 & 53.8 & 0.26 & 5.2 \\
\hline 10 & Chronic pyelonephritis & 53 & $\mathbf{M}$ & 1.70 & 11.2 & 35.6 & 0.31 & 6.3 \\
\hline 11 & Chronic glomerulonephritis & 49 & $\mathbf{M}$ & 1.73 & 10.4 & 25.3 & 0.41 & 8.8 \\
\hline 12 & Chronic glomerulonephritis $\dagger$ & 34 & $\mathrm{~F}$ & 1.53 & 10.0 & 30.5 & 0.33 & 6.9 \\
\hline 13 & Chronic pyelonephritis & 51 & $\mathbf{M}$ & 1.80 & 9.42 & 19.2 & 0.49 & 12.0 \\
\hline 14 & Chronic glomerulonephritis $\dagger$ & 16 & $\mathbf{M}$ & 1.55 & 9.93 & 34.2 & 0.30 & 9.3 \\
\hline 15 & Chronic pyelonephritis $\dagger$ & 39 & $\mathbf{M}$ & 1.73 & 6.08 & 18.5 & 0.33 & 12.8 \\
\hline 16 & Chronic glomerulonephritis $\dagger$ & 27 & $\mathbf{M}$ & 1.68 & 5.93 & 15.4 & 0.38 & 12.4 \\
\hline 17 & Chronic glomerulonephritis $\dagger$ & 57 & $\mathbf{M}$ & 1.65 & 4.33 & 14.2 & 0.30 & 12.6 \\
\hline
\end{tabular}

* GFR = glomerular filtration rate; $\mathrm{Tm}_{\mathrm{glucose}}=$ maximal transport of glucose; $\mathrm{P}_{\mathbf{C r}}=$ plasma creatinine concentration. Values for GFR in the Table represent the mean of the first three to four clearance periods before the infusion of concentrated glucose solutions. Values for $\mathrm{Tm}_{\text {glucose }}$ represent the mean of all values for $\mathrm{T}_{\text {glucose }}$ obtained after glucose reabsorption had reached a plateau. Observations generally were continued until blood sugar levels exceeded $600 \mathrm{mg}$ per $100 \mathrm{ml}$. Values recorded for GFR and $\mathrm{T}_{\text {glucose }}$ are corrected to $1.73 \mathrm{~m}^{2} \mathrm{BSA}$.

$\dagger$ The dagger indicates that diagnosis was established on the basis of renal biopsy. In Patient 5 an autopsy was performed. In other patients, the diagnosis was based on clinical criteria. Patient 9 was studied on two occasions, separated by an interval of 7 months. Between these two studies, his disease progressed according to all criteria, and 14 months after the second study, he died of terminal uremia. An autopsy was performed. In the first experiment, 17 clearance periods were performed; in the second, 21 periods were performed. The difference between GFR in the two studies was highly significant $(p<0.01)$. The glucose titration curve from the initial study showed little splay. The curve from the second study showed an impressive increase in splay.

pear in the urine at blood sugar levels too low to saturate the majority of nephrons. Conversely, in any nephrons with preferential glomerular damage resulting in a reduction in GFR, saturation of tubular reabsorptive capacity for glucose would require blood sugar levels higher than those required to saturate the majority of nephrons. Either type of abnormal nephron would increase the splay in the glucose titration curve, and if both were present simultaneously the effect on splay would be additive.

In the present studies glucose titrations have been performed on 17 patients with chronic progressive renal disease. Glomerular filtration rates ranged from 4.3 to $58.1 \mathrm{ml}$ per minute. The patients selected for study had either chronic pyelonephritis, a disease in which the primary pathologic changes afflict the tubules, or chronic glomerulonephritis, a process characterized by preferential pathologic involvement of glomeruli.

\section{Methods}

Eighteen glucose titration studies were performed on the 17 patients with chronic renal disease, all of whom were judged to be in optimal salt and water balance. The diagnosis in each patient was established either by renal biopsy or necropsy or on the basis of clinical criteria considered by at least three different observers to be unequivocal (Table I). Each patient elected to take part in these studies after being informed fully of their investigative nature, the details of the procedures, and possible untoward effects. All drugs except digitalis were withheld for at least 24 hours before study. Food was withheld for 8 hours, but ad libitum water intake was permitted. Patients with GFR in excess of $10 \mathrm{ml}$ per minute were encouraged to drink approximately a liter of water beginning 90 minutes before the first clearance period. Patients with lower GFR received smaller amounts of water.

Glomerular filtration rate was determined by the clearance of carboxyl-labeled inulin- ${ }^{14} \mathrm{C}$. A priming dose of inulin ${ }^{14} \mathrm{C}$ was administered intravenously and a sustaining dose delivered by constant infusion throughout the duration of the studies. No patient received more than $50 \mu \mathrm{c}$ of ${ }^{14} \mathrm{C}$ during the course of a titration study. After an equilibration period of approximately 45 minutes, three or more control clearance periods were obtained; no glucose was infused during this interval. Thereafter, plasma glucose levels were elevated progressively by increasing the concentration of glucose in the infusate in the following sequence: $10 \%, 15 \%, 20 \%$, and $50 \%$. All solutions were infused at a rate of $4 \mathrm{ml}$ per minute. In- 


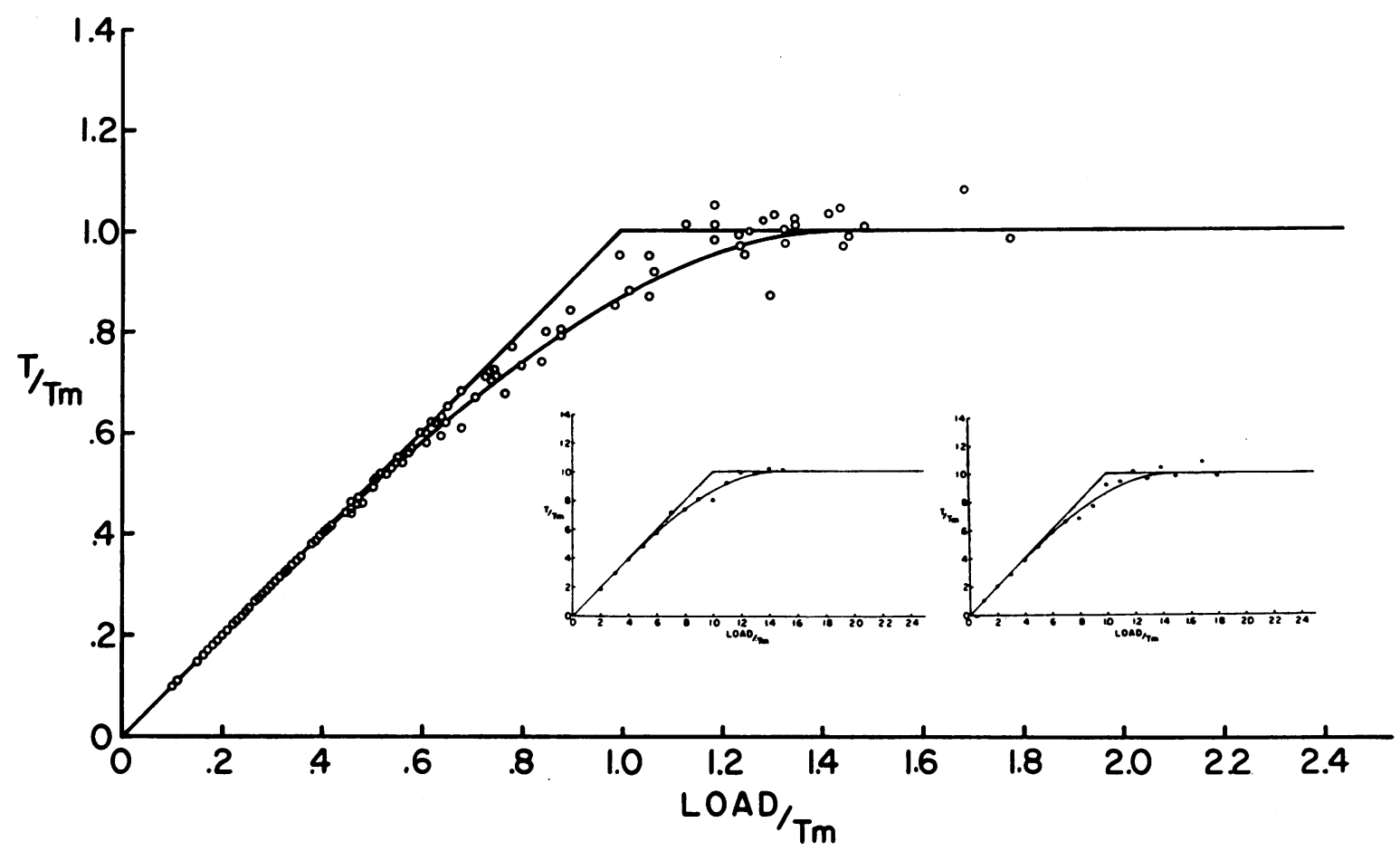

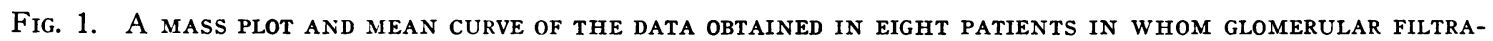
TION RATES (GFR) EXCEEDED 15 ML PER MINUTE PER $1.73 \mathrm{~m}^{2}$ BSA. T refers to glucose reabsorption in milligrams per minute. Load refers to the filtered load of glucose calculated as GFR $\times$ plasma glucose concentration. Both terms are factored by maximal rate of transport $(\mathrm{Tm})$ to allow data from different patients to be plotted on the same graph. The two plots shown in the inserts are the mean curves for the patients with GFR levels between 16.6 and $22.7 \mathrm{ml}$ per minute (left) and 43.8 and $58.1 \mathrm{ml}$ per minute (right), respectively.

dividual clearance periods varied from 10 to 20 minutes in duration depending upon the rate of urine flow. The number of periods obtained with each infusion varied from 2 to 5 , and the total number of periods per study ranged from 15 to 22 . Blood and urinary glucose concentrations were followed semiquantitatively during the infusions with glucose oxidase paper.

Arterial blood was collected throughout each clearance period from either the radial or brachial artery via an inlying Cournand needle. By virtue of the continuous collection technique employed, each plasma glucose level represents an integrated value for the entire clearance period. Blood samples were centrifuged shortly after collection, and all analyses were performed on the day of the studies. Urine was collected via an indwelling urethral catheter. All catheterizations were performed by a physician, and rigid aseptic technique was followed. Neosporin ointment was used as a lubricant. Urine cultures were obtained routinely at intervals after completion of the studies; there was no evidence that bacteriuria had been initiated in any of the patients. Each urine collection was terminated by two bladder washouts consisting of $20 \mathrm{ml}$ of distilled water followed by one or more injections of air.

For the determination of inulin- ${ }^{14} \mathrm{C}$, plasma samples were deproteinized with zinc sulfate, and $0.5-\mathrm{ml}$ samples of the filtrate were pipetted in duplicate into a toluenebased scintillation fluid (2). One $\mathrm{ml}$ of urine diluted $1: 5$ or $1: 10$ was pipetted in duplicate into the scintillation solution. All samples were counted for at least 10,000 counts in a Packard Tri-Carb liquid scintillation spectrometer (model 3214). A ${ }^{14} \mathrm{C}$ standard was added to each sample, and recoveries were determined in order to correct for quenching. Glucose concentration in plasma and urine was determined by a glucose oxidase method (3). Plasma proteins were precipitated with $0.3 \mathrm{M}$ perchloric acid, and analyses were performed on the supernatant. Urine samples were passed through a column containing anion and cation exchange resins (Amberlite 120 and 400 ) before analysis to remove interfering compounds. Distilled water was used as a wash. Recovery experiments done on urine passed through these columns showed no loss of glucose as long as an adequate amount of wash was used.

Glucose titration curves and frequency distribution curves were calculated according to the technique originally described by Smith and associates (1) and recently re-presented by Letteri and Wesson (4). The value for $\mathrm{Tm}$ in each experiment was calculated by averaging the values for glucose reabsorption $\left(T_{\text {glucose }}\right)$ during rising blood sugar concentrations after glucose reabsorption had reached a plateau. In most studies, measurements were 


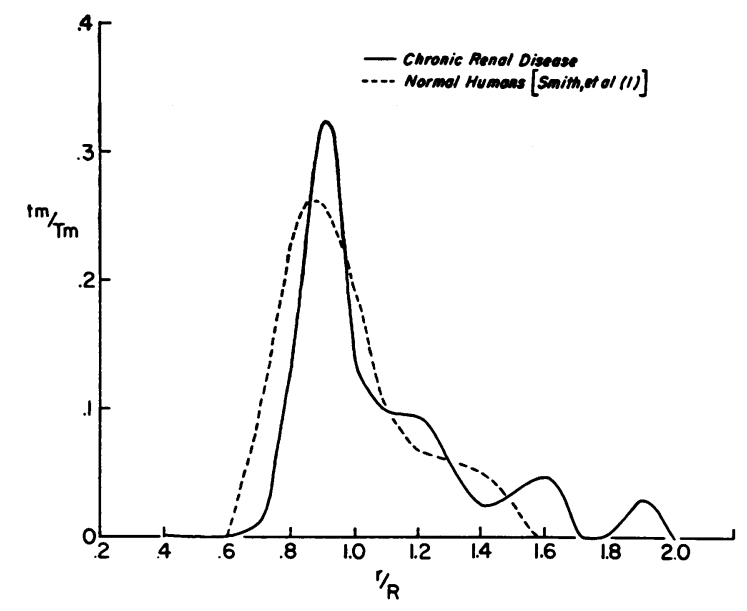

Fig. 2. The FReQUency distribution CURVE for the DATA DEPICTED IN FiguRE 1 COMPARED WITH A SIMILAR CURVE obtained by SMith and associates (1) on NORMAL INDIVIDUALS. $\mathrm{tm} / \mathrm{Tm}$ represents the rate of glucose reabsorption in saturated nephrons expressed as a fraction of the $\mathrm{Tm}$ values for both kidneys considered as a unit; thus this term serves as an index of the fraction of the total nephron population. $r / R$ is an expression for the ratio of glomerulotubular balance of groups of nephrons $(r)$ to the mean value for glomerulotubular balance of both kidneys as a unit $(R)$. The frequency distribution curve, therefore, depicts the relative frequency of nephron groups with various levels of glomerulotubular balance related to the mean value for the two kidneys.

continued until the blood sugar concentrations were well in excess of $600 \mathrm{mg}$ per $100 \mathrm{ml}$. Some variation in the individual values for glucose reabsorption occurs at blood sugar concentrations above the level at which Tm occurs; hence values for $\mathrm{T} / \mathrm{Tm}$ fall above as well as below the line of unity in titration curves. Individual titration curves were constructed by fitting the best curve to the experimental points.

\section{Results}

Values for GFR, $\mathrm{Tm}_{\text {glucose, }}$ and GFR/Tm $\mathrm{T}_{\text {glucose }}$ ratios are shown in Table $\mathrm{I}$. Glomerular filtration rate ranged from 4.3 to $58.1 \mathrm{ml}$ per minute per $1.73 \mathrm{~m}^{2} \mathrm{BSA}$.

A mass plot of the glucose titration data for all patients with GFR values of over $15 \mathrm{ml}$ per minute is shown in Figure 1. In none of these patients was splay marked, and the mean curve does not differ appreciably from that observed by Smith and his co-workers in normal individuals (1). Of the eight patients included in this group, GFR ranged from 16.6 to $22.7 \mathrm{ml}$ per minute in four and from 42.3 to $58.1 \mathrm{ml}$ per minute in the other four. Before including these patients in the same population, we calculated the mean titration curves separately for the two groups. These are presented as inserts in Figure 1. The two curves are identical.

Figure 2 depicts the frequency distribution curve, calculated according to the technique employed by Smith and co-workers (1), for the titration data shown in Figure 1. The curve closely resembles that obtained by Smith for normal man (1). The latter is included in the Figure for comparison.

In Figure 3, a mass plot is shown for the titration data obtained in the four patients with GFR values between 10 and $15 \mathrm{ml}$ per minute. Each of the individual titration curves exhibited an exaggerated splay, and the mean curve leaves the theoretical line earlier, returns to it later, and shows considerably more splay than does the curve depicted in Figure 1.

In Figure 4, data are shown for patients in whom GFR was below $10 \mathrm{ml}$ per minute. The mean curve leaves the theoretical line at a load of less than $20 \%$ of $\mathrm{Tm}$, and a load of approximately twice $\mathrm{Tm}$ was required to saturate the reabsorptive capacity. The splay in the mean curve is very pronounced.

\section{Discussion}

The present studies were performed in an effort to examine the level of nephron homogeneity in a functional context in patients with chronic renal disease. The glucose titration test was employed in this quest. This technique measures the relationship between glomerular filtration rate and glucose reabsorptive capacity in the composite population of urine-forming nephrons in both kidneys. If the structural heterogeneity that characterizes the kidney with advanced renal disease produces selective and uncompensated functional impairment in either glomerular filtration rate or glucose reabsorptive capacity in segments of the nephron population, the glucose titration curve might reveal this. As already noted, if there are any nephrons in a kidney in which glucose reabsorption is defective but GFR is not diminished correspondingly, glucose reabsorptive capacity should be exceeded at a plasma glucose concentration below that which will saturate the majority of nephrons. Thus glucose will be excreted by 


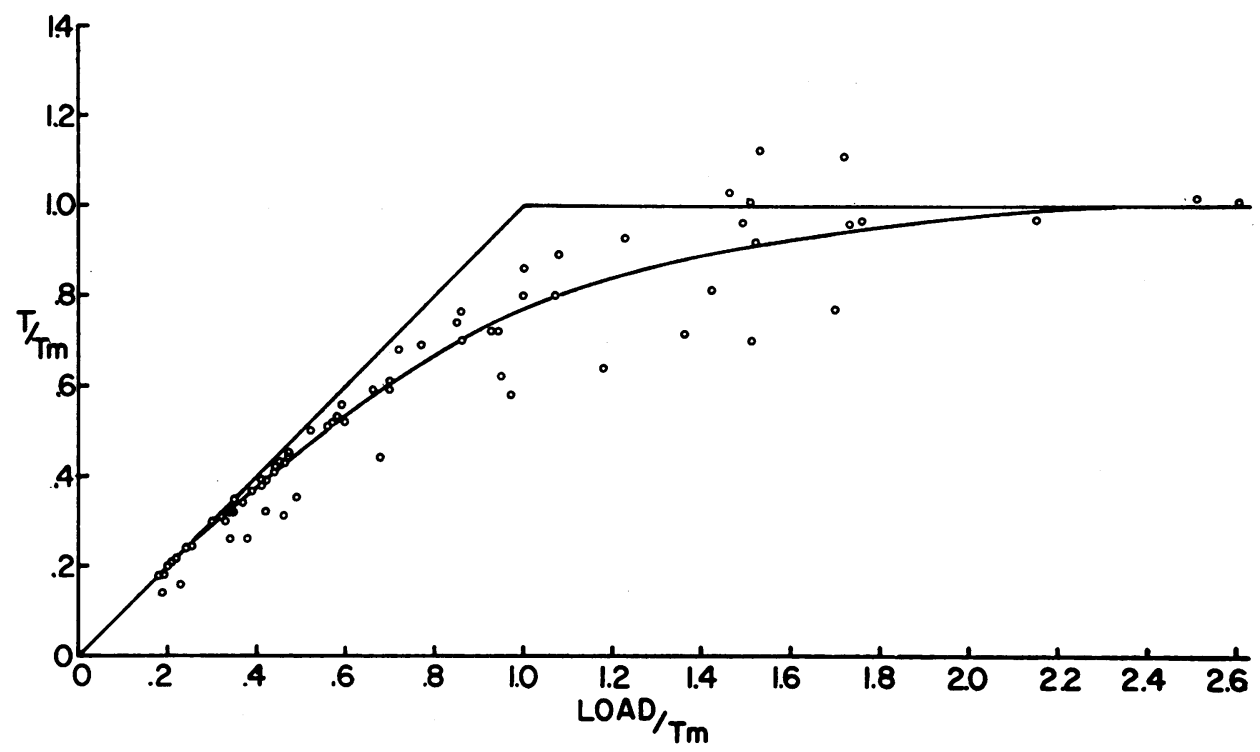

Fig. 3. A mass plot and mean curve of the data obtained in patients with GFR values BETWEEN 10 AND 15 ML PER MINUTE PER $1.73 \mathrm{~m}^{2}$.

these units when the filtered load of glucose is well below the $\mathrm{Tm}$ level. On the other hand, if there are any nephrons with glomerular damage and reduced filtration rate but unimpaired tubular reabsorptive capacity for glucose, continuing glucose reabsorption would occur until blood sugar levels were well in excess of those required to saturate the majority of nephrons. This would be reflected in the titration curve by the achievement of Tm only after filtered load values exceed Tm appreciably. In designing these studies, we chose to include patients with both pyelonephritis and glomerulonephritis because the former disease is primarily an interstitial abnormality that can produce preferential tubular damage, whereas the latter is characterized by preferential anatomic alterations in glomeruli. The data then were pooled and mean titration curves constructed. The absence of an abnormal pattern of splay in such group data would suggest an absence of marked heterogeneity of glomerulotubular balance, with respect to glucose; the presence of splay could indicate the existence of functional heterogeneity. Splay, however, might also have a kinetic explanation independent of the level of homogeneity of glomerulotubular balance.

We believe that two aspects of the data are of considerable interest. First, exaggerated splay was not observed in patients with glomerular fil- tration rates above $15 \mathrm{ml}$ per minute. Second, an increase in splay was observed in patients with GFR values below $15 \mathrm{ml}$ per minute, and in those in whom GFR was less than $10 \mathrm{ml}$ per minute the splay was markedly exaggerated.

The lack of exaggerated splay in patients with GFR above $15 \mathrm{ml}$ per minute

In the patients included in Figure 1, glomerular filtration rate, in relation to predicted normal values, was reduced by from $50 \%$ to over $85 \%$. Presumably, the reduction in nephron population, particularly in the patients with pyelonephritis, was at least as great as the reduction in GFR (5), yet, as indicated, the splay in these patients did not differ appreciably from that observed in normal man. Moreover, there was no greater splay in patients with GFR ranging from 16.6 to $22.7 \mathrm{ml}$ per minute than in those with GFR ranging from 42.3 to $58.1 \mathrm{ml}$ per minute, nor was the splay any different in the patients with pyelonephritis than in those with glomerulonephritis.

One possibility that must be considered in interpreting these data is that the nephrons with glomerular damage and those with tubular damage were equally distributed and that the one category of defective nephron canceled the effects of the other on the glucose titration curve. However, 


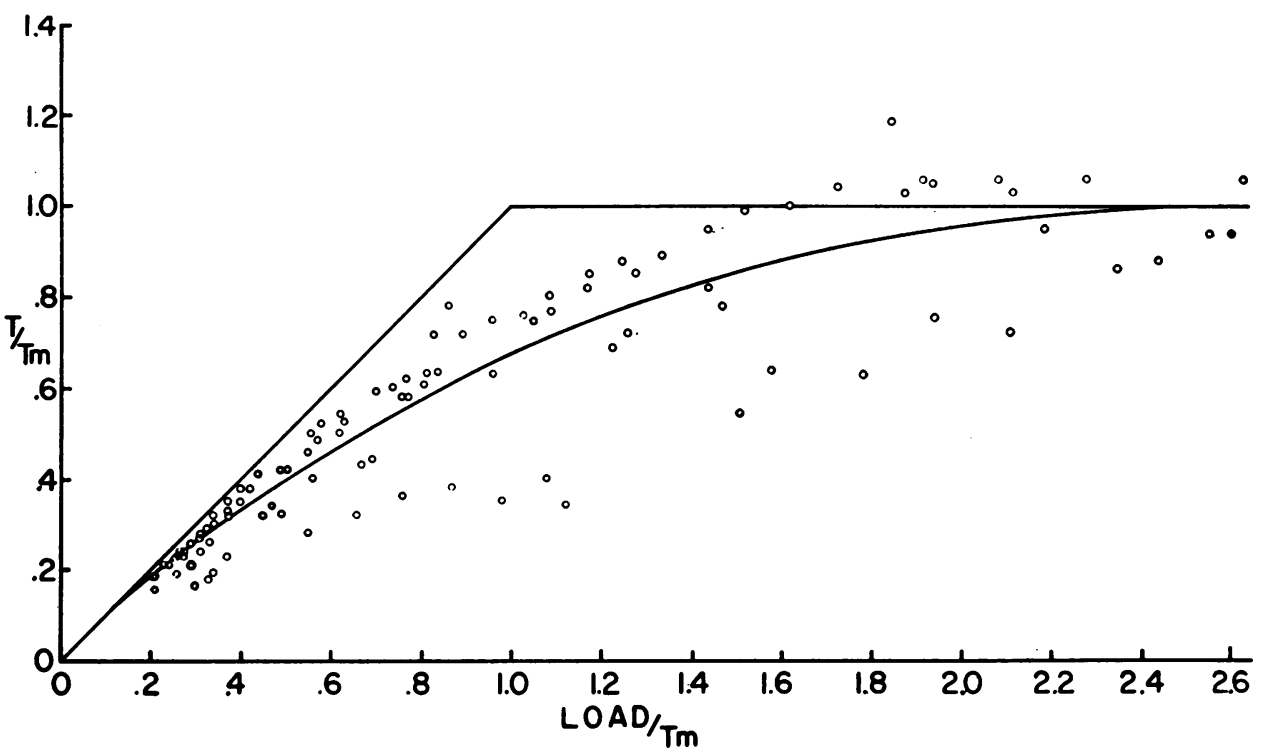

Fig. 4. A mass plot and MEAN CURVe for the data from patients with GFR values BELOW $10 \mathrm{ML}$ PER MINUTE PER $1.73 \mathrm{M}^{2}$.

the nature of the glucose titration test would seem to exclude this possibility, inasmuch as each type of abnormal unit would contribute separately to splay and if both types were present simultaneously splay would be exaggerated rather than obscured. The results do not exclude the existence of tubular damage that does not impair glucose reabsorptive capacity or of glomerular damage that does not decrease GFR in the involved nephrons. On the other hand, if part of the urine-forming nephrons had either defective glucose reabsorptive capacity or diminished GFR, the experimental data would require a proportional decrease in filtration rate or glucose reabsorption, respectively, in the involved nephrons.

The emergence of exaggerated splay in patients with GFR below $15 \mathrm{ml}$ per minute

There are at least three explanations that may be entertained for the progressive increase in splay observed in patients with GFR values below 15 $\mathrm{ml}$ per minute.

A) Functional heterogeneity secondary to far advanced disease. It is conceivable that at very low levels of GFR, the percentage of abnormal nephrons increases so that the pool of urine-forming nephrons constitutes an increasingly heterogeneous population. If this is valid, an explana- tion must be sought for the absence of exaggerated splay in the patients in whom GFR levels were reduced by as much as $85 \%$.

B) Heterogeneity due to functional adaptations. Asymmetric hypertrophy of glomeruli and tubules could convert a nephron population into a heterogeneous group irrespective of the degree or severity of anatomic damage to the urine-forming nephrons. For example, if scar tissue within the severely diseased kidney restricted the degree to which GFR could increase adaptively in some areas of the kidney and the degree to which tubules could hypertrophy in other areas, heterogeneity might well appear. Again it would be necessary to determine why splay emerged only in those patients with GFR levels below $15 \mathrm{ml}$ per minute rather than appearing in a gradually increasing pattern that paralleled the decrement in nephron population.

C) Alterations in kinetics of glucose transport. As recently reviewed by Berliner (6), glucose reabsorption may be compared to a standard enzymatic reaction. Indeed if filtered glucose is employed as the substrate term and the rate of glucose reabsorption as the reaction velocity term, glucose reabsorption may be characterized by the Michaelis-Menten equation for analyzing the kinetics of enzyme reactions. Splay in the titration curve is analogous to the combination of first-order and zero-order kinetics that produces splay in 
a typical Michaelis-Menten plot. If this analogy is extended, it is evident that a change in the dissociation constant of a glucose : carrier complex, the presence of competitive inhibitors, or a change in the fraction of total glucose molecules colliding with the carrier (due to a change in tubular diameter or velocity of flow) could influence the titration curve. Splay thus could increase without an underlying change in functional homogeneity of the constituent nephrons, yet in this explanation as in the previous two, a basis must be found for the fact that splay emerged only at very low GFR.

The present studies do not allow differentiation among the three possibilities presented. In an effort to obtain further insight into the mechanism of the splay, we have employed an experimental model and the results of the latter studies will be described in a separate communication (7).

\section{References}

1. Smith, H. W., W. Goldring, H. Chasis, H. A. Ranges, and S. E. Bradley. The William Henry Welch Lectures. II. The application of saturation meth- ods to the study of glomerular and tubular function in the human kidney. J. Mt Sinai Hosp. 1943, 10, 59.

2. Beaven, M. A., and R. R. Maickel. Stereoselectivity of norepinephrine storage sites in heart. Biochem. biophys. Res. Commun. 1964, 14, 509.

3. Bergmeyer, H. U., and E. Bernt. D-Glucose determination with glucose oxidase and peroxidase in Methods of Enzymatic Analysis, 1st ed., H. U. Bergmeyer, Ed. New York, Academic Press, 1963 , p. 123.

4. Letteri, J. M., and L. G. Wesson, Jr. Glucose titration curves as an estimate of intrarenal distribution of glomerular filtrate in patients with congestive heart failure. J. Lab. clin. Med. 1965, 65, 387.

5. Bricker, N. S., S. Klahr, and R. E. Rieselbach. The functional adaptation of the diseased kidney. I. Glomerular filtration rate. J. clin. Invest. 1964, 43, 1915.

6. Berliner, R. W. Outline of renal physiology in Diseases of the Kidney, M. B. Strauss and L. G. Welt, Eds. Boston, Little, Brown, 1963, p. 30.

7. Shankel, S. W., A. M. Robson, and N. S. Bricker. On the mechanism of the splay in the glucose titration curve in advanced experimental renal disease in the rat. J. clin. Invest. 1967, 46, 164.

\section{SPECIAL NOTICE TO SUBSCRIBERS}

Post Offices will no longer forward the Journal when you move.

Please notify The Journal of Clinical Investigation, Business Office, 10 Stoughton Street, Boston, Mass. 02118, at once when you have a change of address, and do not omit the $\mathrm{Zip}$ Code number. 